\title{
Incremento de los riesgos geomorfológicos en el espacio urbano de Sarajevo como consecuencia del proceso de suburbanización y las políticas de neoliberalización
}

\section{Increase of geomorphological risks in the urban space of Sarajevo as a consecuence of the process of suburbanisation and policies of neoliberalisation}

\author{
Jordi Martín-Díaz, Jordi Nofre, Marc Oliva y Pedro Palma ${ }^{1}$
}

\begin{abstract}
RESUMEN
El pasado mes de mayo de 2014 , las precipitaciones asociadas al profundo sistema de bajas presiones Tamara provocaron severas inundaciones y numerosos deslizamientos de tierra que afectaron a vastas áreas de la región balcánica occidental y central, alcanzando también la ciudad de Sarajevo (Bosnia y Herzegovina). En este episodio quedaron evidenciados los riesgos hidrológicos y geomorfológicos que afectan actualmente a un número significativo de áreas urbanas y suburbanas construidas en la capital bosnia desde el fin de la guerra. A partir de un trabajo observacional y etnográfico realizado entre los años 2010 y 2013, y con apoyo operacional de los Sistemas de Información Geográfica, este artículo tiene como objetivo analizar la creciente insostenibilidad urbana de la ciudad de Sarajevo, producida tanto por una densificación del fondo de valle en áreas inundables así como el proceso de suburbanización que ha ve-
\end{abstract}

1 Jordi Martín-Díaz, Departamento de Geografía Humana, Universidad de Barcelona, jordi.martin@ub.edu. Jordi Nofre, Centro de Estudos de Sociologia-UNL (CesNova), Faculdade de Ciências Sociais e Humanas, Universidade Nova de Lisboa, jnofre@fcsh.unl.pt. Marc Oliva, Centro de Estudos Geográficos, Instituto de Geografia e Ordenamento do Território Universidade de Lisboa, oliva_marc@yahoo.com. Pedro Palma, Centro de Estudos Geográficos, Instituto de Geografia e Ordenamento do Território, Universidade de Lisboa, p.palma@campus.ul.pt. 
nido produciéndose principalmente en las laderas de los macizos que abrigan la ciudad. Estos procesos son, respectivamente, resultado de la implementación de las políticas de liberalización económica y de la necesidad por parte de las personas refugiadas en Sarajevo de estabilizar su situación en la ciudad tras el conflicto bélico.

PAlabras ClaVe: riesgos geomorfológicos; suburbanización; desarrollo urbano; neoliberalización; ciudades post-socialistas.

\section{AbSTRACT}

In May 2014, the rainfall associated with the deep low pressure system called Tamara caused severe flooding and numerous landslides in the Western Balkans region, also reaching the city of Sarajevo (capital of Bosnia and Herzegovina). In this episode hydrological and geomorphological hazards affecting a significant number of urban and suburban areas built in the Bosnian capital since the end of the war were evidenced. From an observational and ethnographic work carried out between 2010 and 2013 and operational support of GIS, this paper aims at highlighting the unsustainable direction of its built environment. This has been caused by the densification occurred in floodplain as well as the intense suburbanisation process of the slopes. Both processes are respectively produced by the international policies promoting a neoliberal urban development and the need of people refugeed in Sarajevo to stabilise their situation in the city after the war.

KEY WORDS: geomorphological hazards; suburbanisation; urban development; neoliberalisation; post-socialist cities.

\section{INTRODUCCIÓN}

Los nuevos paradigmas políticos, económicos y financieros surgidos a lo largo de las últimas décadas, en gran parte asociados al proceso de globalización neoliberal (Peck et al., 2009; Theodore et al., 2011) -o si se prefiere, postindustrial (Hyman, 1999)-, han comportado profundos cambios en el modelo de urbanización en las regiones y países 'occidentales' así como, muy especialmente, en aquéllos de Europa central y oriental que pertenecieron al bloque socialista (Borén y Gentile, 2007; Hegedüsa, 2007; Diener y Hagen, 2013; Sýkora y Stanilov, 2014). En términos de planificación y gestión territorial y urbana, la desaparición del bloque soviético comportó la transición del llamado modelo socialista de urbanización (Tosics, 2005), dando paso a un nuevo modelo que tiende a convergir con el capitalista en la que la privatización del suelo y la introducción del mercado libre en términos de desarrollo urbano y promoción inmobiliaria adquirieron una relevancia muy significativa. 
Así, la readaptación de las formas y estructuras urbanas heredadas de los respectivos regímenes socialistas del siglo xx por parte de los nuevos agentes de producción de espacio urbano -aparecidos al amparo de las nuevas estructuras institucionales y políticas surgidas como resultado del proceso de transición hacia una democracia liberal- se ha desarrollado como resultado de la aplicación de mecanismos y estrategias de planificación urbana y territorial de claro sesgo neoliberal (Harvey, 1989; Brenner y Theodore, 2005; Baeten, 2012; Swyngedouw et al., 2002; Tasan-Kok, 2012; Theodore et al., 2011). Ello adquiere especial importancia, por ejemplo, en la estructuración de los espacios públicos y comunitarios verdes, ya que la propiedad estatal del suelo, de las empresas constructoras y la ausencia de un mercado inmobiliario de régimen libre permitieron un mayor respeto y cumplimiento de los ideales socialistas en materia de urbanismo (Horner, 1978).

De hecho, la mayor compacidad y densidad edificatoria de las áreas urbanas desarrolladas en la segunda mitad del siglo pasado en la mayor parte regiones y países del bloque comunista presentaban un mayor número de hectáreas de espacios comunitarios y verdes en comparación con las ciudades de Europa occidental y meridional (French y Hamilton, 1979; Szelenyi, 1996). En este sentido, las formas y estructuras urbanas surgidas al amparo de los regímenes comunistas de la Europa central y oriental eran consideradas medioambientalmente más sostenibles que el de las ciudades capitalistas (Hirt y Stanilov, 2009).

En el caso de la capital de Bosnia y Herzegovina, Sarajevo, las transformaciones urbanas acaecidas después de la guerra de Bosnia (1992-1995) han favorecido cierta reconfiguración y expansión del territorio urbanizado a través fundamentalmente de procesos de terciarización del suelo urbano en sus áreas centrales y del desarrollo de miles de viviendas dando como resultado un proceso de suburbanización, en términos de Guiseppe Dematteis (1998) y Tony Champion (2001). A su vez, tales transformaciones urbanas en la Sarajevo posbélica han tenido que lidiar con los enormes retos medioambientales que presentaba (y aún presenta) la capital bosnia, en un ambiente político y social caracterizado por la tensión étnico-nacional y político-territorial en frágil equilibrio en términos geopolíticos. Especialmente en lo que se refiere al proceso de reasentamiento de los refugiados y desplazados internos, cabe remarcar que la infraestructura residencial tuvo que adoptarse al difícil y rugoso marco geomorfológico en donde se asienta la ciudad de Sarajevo (figura 1). De ahí que surjan dudas acerca de la sustentabilidad urbana de la ciudad en lo que se refiere a las condiciones en que se ha producido la suburbanización de parte de su territorio bajo políticas de 
planificación y gestión urbana neoliberal, las cuales han tendido a omitir o menospreciar, en el mejor de los casos, la existencia de ciertos riesgos de índole geomorfológica e hidrológica.

La amenaza geomorfológica en entornos urbanos ha sido ampliamente estudiada a lo largo de las últimas décadas desde un amplio abanico disciplinar (Gupta y Ahmad, 1999; Huang y Mu, 2000; Adeli y Khorshiddoust, 2011; de Waele et al., 2011). En el caso de ciudades del Este y Sureste de Europa, un número relevante de artículos han puesto de relevancia el potencial riesgo que algunos tipos de procesos geomorfológicos (como puedan ser colapsos del terreno, procesos de vertiente, inundaciones y avenidas excepcionales) pueden suponer en algunas áreas suburbanas recientemente edificadas y localizadas en ambientes kársticos (Pohl et al., 2004; Stecchi et al., 2012; PapadopoulouVryniotia et al., 2013). Sin embargo, y a pesar de presentar un cuadro similar al expuesto en lo que se refiere a la existencia de ciertos riesgos geomorfológicos tanto en sus áreas suburbanas como en algunas áreas de nueva centralidad desarrolladas recientemente, el caso particular de la ciudad de Sarajevo ha sido poco tratado contando sólo con alguna referencia, como por ejemplo Golobic y Cof (2010).

Este artículo tiene como objetivo identificar tanto las causas que han producido un incremento de los riegos geomorfológicos -deslizamientos de tierra- e hidrológicos en la ciudad de Sarajevo durante el período posbélico así como las áreas urbanas y suburbanas potencialmente afectadas. El análisis de dichos riesgos tendrá en cuenta la aplicación de la agenda ambiental de Sarajevo en el complejo entorno social, étnico y político que ha surgido después de la guerra de Bosnia. A pesar del consenso alcanzado para el desarrollo de Sarajevo a través de estrategias alineadas con la normativa europea para la sostenibilidad, en este artículo se argumenta que la superficie construida en la ciudad ha incrementado su vulnerabilidad como resultado, por una parte, de la necesidad de obtener una vivienda estable por parte de las personas refugiadas en Sarajevo tras los episodios de limpieza étnica ocurridos durante la guerra de Bosnia (1992-1995) así como también por la adopción, por parte de la administración pública local, regional y nacional, del urbanismo neoliberal como paradigma medular del desarrollo post-socialista de la capital bosnia. 
FIGURA 1

LOCALIZACIÓN DE SARAJEVO E IMAGEN AÉREA DE LA CIUDAD

\section{Bosnia and Herzegovina}



\section{Sarajevo}

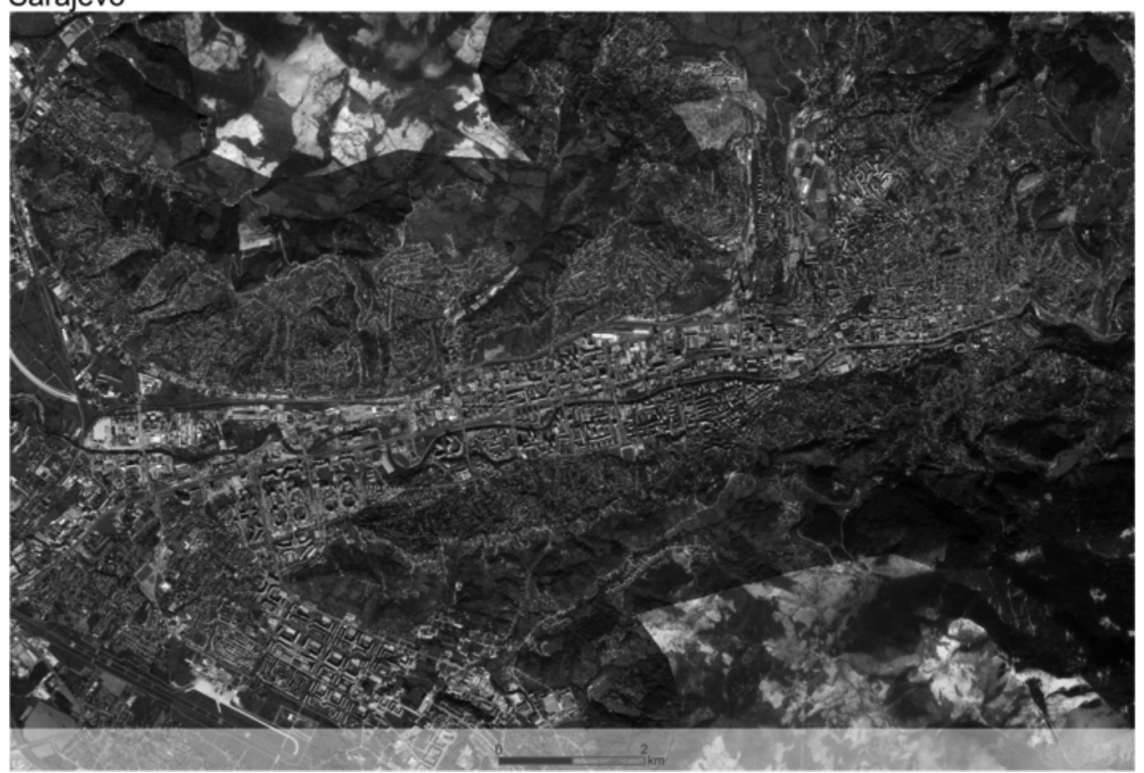

Estudios Geográficos, Vol. LXXVII, 281, pp. 543-567, julio-diciembre 2016 ISSN: 0014-1496, eISSN: 1988-8546, doi: 10.3989/estgeogr.201618 


\section{Metodología}

El marco metodológico general establecido para la elaboración del trabajo que se presenta en este artículo viene definido, como ya ha sido brevemente anunciado en la sección introductoria, por la constante interacción dialéctica entre el cuadro geomorfológico local y la actuación de los diferentes actores tanto nacionales como internacionales en la transformación urbana de Sarajevo durante el periodo 1996-2013. El establecimiento de este marco metodológico vino inducido a partir de las primeras observaciones de carácter exploratorio realizadas antes del primer trabajo de campo de la investigación llevado a cabo entre los meses de febrero y julio del año 2010. Este trabajo de campo tuvo como objetivo identificar las formas y procesos geomorfológicos e hidrológicos que constituyen amenazas para la población y sus bienes de la ciudad de Sarajevo durante el período posbélico. Las primeras hipótesis de trabajo surgidas en el primer trabajo de campo apuntaban al incremento de áreas urbanas y suburbanas con elevado índice de amenaza geomorfológica.

La segunda fase del trabajo de campo se llevó a cabo entre los meses de julio y diciembre de 2013 con el objetivo de analizar las políticas de desarrollo urbano adoptadas por parte de la administración pública local después de la guerra de Bosnia. Esta tarea tuvo como objetivo principal examinar la adopción de nuevas herramientas de planificación urbana por parte de la misma administración pública local en consonancia con el rol de los actores internacionales en la implementación de la agenda de construcción de la paz liberal. Para verificar la información recopilada a través del trabajo de campo observacional y para analizar qué grado de implicación tienen los diferentes actores institucionales nacionales e internacionales en la producción de nuevos espacios urbanos y suburbanos que presentan amenaza geomorfológica, fueron realizadas un total de 27 entrevistas semi-estructuradas entre los años 2010 y 2013 tanto con técnicos como con responsables de proyectos urbanísticos del Departamento de Planificación Urbana de la Ciudad de Sarajevo, el Instituto de Planificación y Desarrollo Urbano de Sarajevo, la Agencia de Desarrollo Económico y Regional del Cantón de Sarajevo, así como personal de la organizaciones internacionales tales como la Oficina del Alto Representante, responsable de supervisar la implementación de los Anejos civiles de los Acuerdos de Paz. Por motivos de confidencialidad, todas las entrevistas han estado transcritas y archivadas en soporte digital aislado.

En términos de análisis cartográfico cabe señalar que las áreas urbanas y suburbanas de la ciudad de Sarajevo que presentan riesgo de inundaciones y de remociones en masa (ver Alcántara, 2000) han sido cartografiadas mediante los 
Sistemas de Información Geográfica (ArcGIS 10). Los resultados del trabajo observacional preliminar fueron corroborados a través de la identificación, mediante las herramientas de análisis espacial de ArcGIS10 de aquellas áreas urbanizadas con pendientes comprendidas entre 1) el 20\% y el 30\%; y 2) por encima del $30 \%$, porcentaje considerado como crítico para la seguridad de una edificación unifamiliar en lo relativo a potenciales corrimientos de material sedimentario argiloso en pendientes inestables (Easterbrook, 1999). Además de la identificación de vertientes inestables mediante ambas técnicas citadas, se cartografiaron aquellas áreas susceptibles de ser afectadas por inundaciones debido a las crecidas del río Miljacka a lo largo de su tramo urbano. Como nivel de referencia se adoptó el valor 2,5 m. que es el nivel que se alcanzó en el episodio de inundaciones del pasado mes de mayo de 2014 (Sarajevo Times, 2014) y que corresponde al episodio de inundaciones más importante en los Balcanes Occidentales registrado en los últimos 120 años (Agencias, 2014).

Con todo, cabe señalar que la producción cartográfica presentada en este artículo contó con ciertos contratiempos. Fruto de la inexistencia de un convenio público entre las universidades públicas y las agencias públicas de gestión del territorio en Bosnia y Herzegovina, no existe ningún tipo de servidor público de bases cartográficas ni productos derivados, tales como Modelos Digitales Terrestres, que permitan un posterior análisis cartográfico con plenas garantías funcionales y científicas. Es por ello que se optó por recurrir a los datos de elevación disponibles en fuentes abiertas internacionales de información geográfica, las cuales presentan una resolución de $30 \mathrm{~m}$. En el caso particular de la ciudad de Sarajevo y su región metropolitana, los datos provienen del Advanced Spaceborne Thermal Emission and Reflection Radiometer, usado comúnmente en la elaboración de cartografía geomorfológica (Kääb et al., 2003; Kamp et al., 2005).

\section{El CONTEXTO GEOMORFOlÓGICO E HIDROGRÁFICO DE LA CIUDAD DE SARAJEVO}

Sarajevo se localiza en una depresión tectónica circundada por los Alpes Dináricos, que se alzan en las inmediaciones con alturas que sobrepasan los $2.000 \mathrm{~m}$ (Treskavica, $2.088 \mathrm{~m}$; Bjelašnica, $2.067 \mathrm{~m}$ ). Este macizo está constituido por rocas sedimentarias de edad secundaria y terciaria, básicamente calizas y dolomías. En el caso particular de Sarajevo, ésta se distribuye en una llanura aluvial alargada a alturas de 500-600 m, con macizos calizos de $1.000-$ $1.500 \mathrm{~m}$ circundando la ciudad. El relieve se organiza en una serie de umbrales rocosos escalonados en altura en torno al fondo de valle, siguiendo la red de fallas principal mientras que los ríos Miljacka y Bosna drenan la cuenca hi- 
drográfica de Sarajevo-Zenica, de edad Terciaria (Hrvatovič, 2005). El relleno sedimentario de la depresión de Sarajevo está constituido por sedimentos cuaternarios que recubren el sustrato de rocas miocénicas. En este sentido, y tal y como sucede en extensas zonas de la Península Balcánica, en esta región se distribuye una amplia variedad de procesos y formas kársticas. El contacto entre el material carbonatado (calizas) y el agua (de precipitación, escorrentía o subterránea) inicia el proceso de disolución del sustrato original (Jennings, 1985). En los alrededores de Sarajevo los procesos kársticos son muy intensos y han originado una amplia amalgama de morfologías kársticas. Como ejemplo de ello, las depresiones cerradas que pueblan en mayor o menor medida la región constituyen áreas con sistemas de drenaje internos que en ambientes kársticos reciben el nombre de poljes (Sweeting, 1973) (ver figura 2). Sus dimensiones oscilan entre decenas de metros y extensiones kilométricas. A su vez, en la llanura aluvial de Sarajevo, también destaca la presencia de otras geoformas kársticas de dimensiones métricas, tales como las dolinas.

FIGURA 2

\section{ESBOZO GEOMORFOLÓGICO DE LA CONFIGURACIÓN REGIONAL DE SARAJEVO}



En el interior de la ciudad la mayoría de estas morfologías han quedado enmascaradas por las edificaciones. Sin embargo, la toponimia local atestigua la abundancia de geoformas kársticas. Así, por ejemplo, algunos nombres de calles presentan la partícula «Dolina» así como lugares como Bijelo Polje, Skrbino Polje, Lasicko Polje, Grlicko Polje, Gornje Telalovo Polje o Poljine confirman el contexto litológico kárstico en el que se asienta la capital bosnia. 
En este sentido, cabría mencionar el hecho de que en lenguaje Serbio/Bosnio/Croata el térmico «polje» es polisémico: a la vez que se usa como unidad geomorfológica, también se emplea para definir el campo, lo que refleja la intrínseca relación entre geoforma y paisaje en la región balcánica. Ambos términos quedan también fuertemente determinados por el discurrir del río Miljacka el cual, después de cruzar un desfiladero calizo en el límite oriental de Sarajevo, vira bruscamente su rumbo con una morfología meandriforme en su primer tramo urbano, formando, ya a partir de este primer tramo urbano del río, una llanura aluvial alargada paralela al curso fluvial.

El contacto entre la depresión de Sarajevo y el relieve circundante es marcado, especialmente en el margen izquierdo del río Miljacka, donde adquiere mayor verticalidad. Por el contrario, el declive de las vertientes del margen derecho es más atenuado. No obstante, la concentración de asentamientos urbanos en ambas vertientes es elevada. Sin duda, la litología y la compleja orografía del terreno condicionan el contexto geomorfológico, que a su vez presenta implicaciones directas en la distribución de la población a lo largo del valle del Miljacka (figura 3). Con una densidad de población visiblemente menor que en el fondo de valle, las casas unifamiliares de entre dos y tres plantas aparecen dispuestas escalonadamente en las vertientes, dejando cierta distancia entre ellas. Como se analizará en los próximos epígrafes, muchas de ellas son resultado del intenso proceso de suburbanización producido por el reasentamiento de desplazados internos posterior a la resolución del conflicto bélico de mediados de la década de1990.

\section{FIGURA 3}

\section{DISTRIBUCIÓN DE LAS VIVIENDAS EN ESCALONES SECUENCIALES CON JARDINES ENTRE MEDIO}
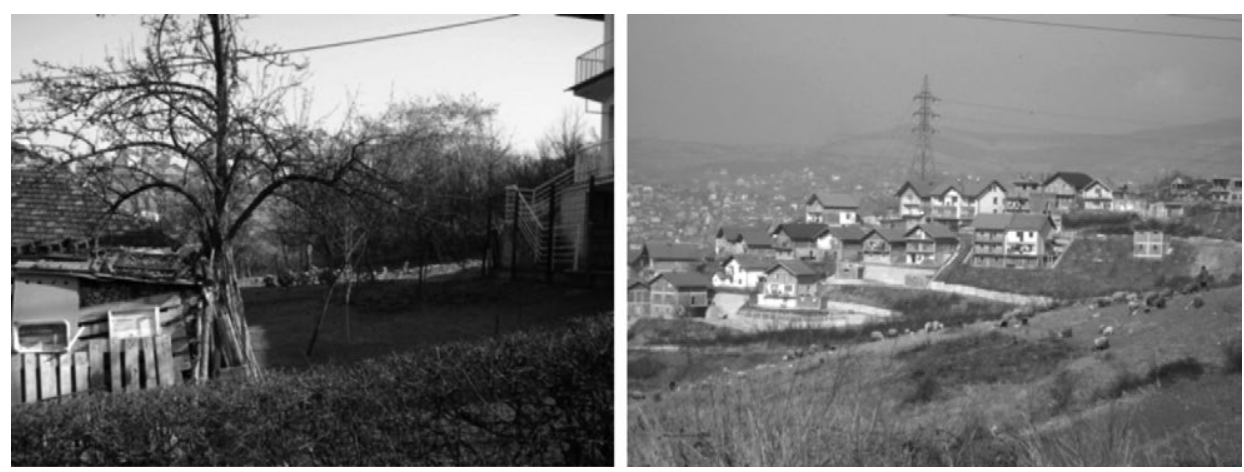

Estudios Geográficos, Vol. LXXVII, 281, pp. 543-567, julio-diciembre 2016 ISSN: 0014-1496, eISSN: 1988-8546, doi: 10.3989/estgeogr.201618 
Volviendo al contexto hidrográfico de la capital bosnia, durante los últimos milenios las inundaciones del río Miljacka han depositado sedimentos en los márgenes del río, que han contribuido a fertilizar el suelo, favoreciendo así las actividades agrícolas tradicionales y la progresiva urbanización e industrialización del territorio. Ello ha comportado sin duda alguna el surgimiento de puntuales situaciones de riesgo acontecidas especialmente en episodios avenidas de fluviales. De hecho, el progresivo aumento de ocurrencia de inundaciones presenta una componente natural, puesto que la mayor variabilidad climática registrada durante los últimos siglos en el Hemisferio Norte ha venido traduciéndose en una mayor frecuencia de las olas de frío e inundaciones en Europa Central y Oriental (Bradley et al., 1993; Luterbacher et al., 2002, 2004). Ello llevó a una intensificación de la tala de árboles anteriormente abundantes en el lecho de inundación del río Miljacka con el objetivo de responder al incremento de la demanda de consumo de combustible leñoso para calefacción de uso doméstico y cocina ${ }^{2}$. Sin embargo, el aumento el proceso de expansión urbano experimentado por la ciudad de Sarajevo especialmente desde tiempos de dominación austro-húngara así como la mayor frecuencia y magnitud de las inundaciones durante el siglo XVIII en la región balcánica (Xoplaki et al., 2001; Wanner et al., 2004) condicionaron la canalización del río Miljacka (1900-1913) con la finalidad de reducir los destrozos causados por las inundaciones (Donia, 2006). El sistema se usa aún hoy en día y consiste en una serie de escalones que reducen la velocidad de flujo y la capacidad de transporte de sedimentos. Los laterales del río fueron sustituidos por áreas urbanizadas que recientemente han sido ocupadas por áreas de nueva centralidad urbana tal y como se expondrá en la segunda mitad de este texto.

De hecho, la ciudad se distribuye a lo largo de una depresión drenada por el río Miljacka que discurre de ENE-WSW, alcanzando su máximo caudal anual entre octubre y diciembre, tal y como sucede en otros ríos bajo influencia climática mediterránea. El Miljacka fija cabecera en una región kárstica (Kadino Selo) y aguas abajo recoge las aguas de los afluentes que descienden bruscamente de las montañas que rodean la capital de BiH. En tan sólo 15 $\mathrm{km}$ desciende más de $1.000 \mathrm{~m}$ de altura (7,3\% de pendiente), lo que incre-

\footnotetext{
${ }^{2}$ Un proceso similar aconteció durante la guerra de Bosnia, cuando los sarajevitas cortaron los árboles para usarlos como combustible leñoso (una vez que el suministro de gas fue cortado), crear espacios para enterrar a los miles de fallecidos por causa de la guerra y generar nuevos espacios para la producción agrícola autosuficiente en una ciudad que fue sitiada durante tres años (Admir et al., 2014).
} 
menta su poder erosivo en el tramo alto de la cuenca y la efectividad del transporte de sedimentos aguas abajo. Todo ello en un ambiente kárstico caracterizado por la alta porosidad del sustrato, hecho que aumenta el potencial destructivo de las potenciales avenidas fluviales. El régimen climático local está condicionado por la influencia continental de centro-Europa y el influjo marítimo del mar Adriático. La condensación del aire húmedo y cálido proveniente del Adriático al superar las barreras montañosas costeras eleva las precipitaciones anuales a valores de $900 \mathrm{~mm}$ anuales, mayoritariamente concentrados en primavera y otoño. Además, la propia orografía abrupta de la región de Sarajevo constituye otro factor de relevancia en el incremento de la probabilidad de ocurrencia de precipitaciones torrenciales durante estas estaciones, que pueden causar daños significativos en equipamientos e infraestructuras.

Precisamente, a mediados de mayo de 2014 tuvieron lugar en la zona inundaciones catastróficas que causaron pérdidas humanas y desperfectos en equipamientos, infraestructuras y viviendas (FENA, 2014a, 2014b). El impacto de

\section{FIGURA 4}

INUNDACIÓN EN EL ÁREA OCCIDENTAL DE SARAJEVO, MUNICIPALIDAD DE ILIDŽA, 15 DE MAYO DE 2014



Fuente: Agencia Anadolu. 
las avenidas fue mayor en el sector occidental de la ciudad, donde los ríos Miljacka y Zeljeznica confluyen con el Bosna (figura 4). Sarajevo no fue la única ciudad afectada por este episodio de lluvias torrenciales, que impactó de manera catastrófica las áreas urbanas y rurales del norte de Bosnia. Hasta 3.000 deslizamientos se contabilizaron entre el 14 y el 16 de mayo en todo el país, muchos de ellos en Sarajevo y alrededores (FENA, 2014c; Jukic, 2014a), 27 personas perdieron la vida y 100.000 edificios fueron destruidos o gravemente afectados (Agencias, 2014; Jukic, 2014b).

Si bien la excepcionalidad del episodio protagonizado por el ciclón Tamara (14-18 de Mayo de 2014) en cuanto a la intensidad y duración de la precipitación es indiscutible, no cabe duda que algunos aspectos derivados de los recientes procesos de urbanización de la ciudad de Sarajevo no contribuyeron a mitigar los efectos de las inundaciones y remociones en masa producidos por tal evento meteorológico. En el próximo punto se analizan los objetivos y visiones medioambientales y de sustentabilidad urbana recogidos en el documento «Estrategia de Desarrollo del Cantón de Sarajevo hasta el año 2015». Posteriormente se procederá a presentar tanto los procesos que han venido dificultando la aplicación de estas visiones a lo largo del período posbélico, reflejados en aquellas áreas urbanas y suburbanas desarrolladas con riesgo potencial significativo de ocurrencia de procesos de vertientes y/o inundaciones que actualmente constituyen un porcentaje significativo de la geografía de Sarajevo.

\section{Visualizando la SARAJEVo POST-SOCIALISTA}

La adopción a partir de mediados de los años noventa del siglo pasado de la planificación estratégica urbana, metropolitana y regional en buena parte de las ciudades europeas occidentales tenía como objetivo tratar la creciente incertidumbre del período posfordista (Bryson y Einsweiler, 1987) a través de la adopción de aproximaciones más flexibles de gobernanza urbana (Harvey, 1989). En el caso particular de las ciudades europeas post-socialistas la aprobación de dichos planes implicaba la incorporación de actores privados en la definición de las visiones de desarrollo urbano (Tsenkova, 2007), dando pie a la promoción de programas y prácticas urbanísticas de naturaleza neoliberal (Sailer-Fliege, 1999; Smith Rochovská, 2007; Stenning et al., 2010).

La aprobación en el año 1998 del documento Estrategia de Desarrollo del Cantón de Sarajevo hasta el año 2015 (plan estratégico en adelante), que seguía 
la combinación característica de visiones a largo plazo y acciones a corto plazo (Albrechts, 2004), permitió la definición del futuro desarrollo urbano de la capital de Bosnia y Herzegovina con la supervisión de expertos internacionales. Así, los objetivos principales definidos en el documento fueron la creación de una metrópolis europea, un entorno de negocios rentable y un ambiente agradable para vivir (KS, 2000). En este sentido, el plan estratégico exponía entre sus prioridades hacer de Sarajevo una ciudad equilibrada medioambientalmente, es decir, «agradable para vivir, una ciudad limpia, una ciudad saludable, una ciudad verde, y una ciudad ecológicamente revitalizada» (KS, 2000: 7). Ello mostraba el deseo de mejorar las condiciones ambientales en la ciudad, aunque algunos de sus parámetros más significativos, como la contaminación del aire, habían mejorado como consecuencia de las crisis económica de finales de los años 80 y del colapso de la actividad industrial durante la guerra (Majstorovic et al., 2006).

Por otra parte, los modelos de urbanización neoliberal (Peck et al., 2009) utilizados en la reconstrucción urbana posbélica de Sarajevo y su ulterior desarrollo (Martín-Díaz, 2014) se inscriben en el objetivo recogido en el plan estratégico de erigir la capital bosnia como una ciudad que atraiga flujos de capital (KS, 2000: 6). Teniendo en cuenta que, tras tres años y medio de conflicto, se partía de un contexto de destrucción del tejido productivo local y regional -caracterizado en los años sesenta, setenta y ochenta por una fuerte implantación de la industria metalúrgica, siderúrgica y farmacéutica (Andjelić, 2003)-, la definición del programa de reactivación económica del Cantón de Sarajevo contó muy especialmente con el apoyo de la comunidad internacional encargada de tutelar la aplicación de los Acuerdos de Paz de Dayton. En este sentido, la reconstrucción urbana de la ciudad de Sarajevo pretendía reconfigurar el espacio urbano y social de la capital bosnia para generar un entorno favorable a la atracción de inversión directa extranjera y a la promoción de la emprendimiento local (Martín-Díaz, 2016). Ello contó con el apoyo del Plan de Acción de la Unión Europea "Towards Sustainability" (1993), elaborado principalmente por la Comisión Europea y que tenía como objetivo final la implementación de la Agenda 21 en la capital bosnia (EC, 1993). Además de ello, y aún de más interés si cabe, es que el mencionado Plan de Acción de la UE fomentaba la promoción de la propiedad privada, la privatización de los activos públicos, la desregulación del mercado laboral, la privatización y desregulación del Estado del Bienestar heredado del sistema socialista Yugoslavo y la creación de un mercado de bienes raíces fueran concebidos como factores económicos centrales para el desarrollo urbano post-bélico y post-socialista de la ciudad de Sarajevo (KS, 2000). 
Sin embargo, la transferencia de modelos de planificación entre países sin tener en cuenta las circunstancias locales suele limitar su eficacia (Masser, 1992; Maier, 1994). De hecho, el plan estratégico del Cantón de Sarajevo no estaba incluido en la legislación urbanística nacional, lo que conllevó que la ejecución de su cuerpo propositivo, hasta la fecha, haya sido menor. Si bien el plan estratégico incorporaba la creación de un mercado de bienes raíces y la flexibilización en relación a la construcción de nuevos templos, el plan constituyó una mera continuación algo «renovada» del Plan Urbano de 1984. A pesar de los cambios de la estructura territorial-administrativa de la ciudad derivados de la integración de los municipios del este y del sur en la República Srpska (RS), los proyectos, la estructura parcelaria espacio urbano, los conceptos o los datos estadísticos utilizados para elaborar los planes de regulación y normativa urbana no fueron actualizados a merced de los profundos cambios sociales, culturales, políticos y económicos que había sufrido la ciudad como consecuencia de la guerra.

SUBURBANIZACIÓN POSBÉliCA Y NEOLIBERALIZACIÓN DEL ESPACIO URBANO COMO FACTORES POTENCIADORES DE LOS RIESGOS GEOMORFOLÓGICOS E HIDROLÓGICOS DE SARAJEVO

La reconstrucción urbana posbélica puso en entredicho la elaboración de una estrategia verosímil para la consecución de cierta sostenibilidad urbana medioambiental a medio y largo plazo, conllevando que la capital bosnia fuera hacia una senda caracterizada por la insostenibilidad urbana. En este sentido, la singularidad y extrema complejidad de la gestión del derecho de retorno y posterior realojamiento de los refugiados - con cerca de la mitad de los 4,4 millones de ciudadanos bosnios refugiados o desplazados internos, volumen que significa el mayor registrado en Europa desde la Segunda Guerra Mundial (Hitchcok, 2003)-, hicieron florecer discrepancias substanciales entre la retórica usada en la planificación urbana, metropolitana y regional estratégica del Cantón de Sarajevo. Las visiones medioambientalistas del plan estratégico mencionadas, sin embargo, quedaron debilitadas tanto por la presión demográfica posbélica como por las políticas de liberalización del suelo.

En cuanto a la presión demográfica, tanto refugiados como desplazados internos tenían el derecho de volver a sus casas como establece el Anejo 7 de los Acuerdos de Paz de Dayton (OAR, 1999). Esta provisión de los acuerdos de paz fue quedo confirmada en Febrero de 1998 cuando se firmó la Declaración de Sarajevo con el objetivo de establecer un marco de actuación integral para 
acoger el retorno de refugiados y desplazados internos a la capital de Bosnia (OAR, 1998). Un aspecto clave del tanto el Anejo 7 como de la Declaración de Sarajevo era la reposesión de la vivienda por parte de las personas que abandonaron la ciudad durante el conflicto. La reposesión de la vivienda, sin embargo, implicaba el desalojamiento de grupos vulnerables de población que durante la guerra habían ocupado las casas y apartamentos abandonados huyendo de los episodios de limpieza étnica. Pero el proceso de reposesión de la vivienda se llevó a cabo sin que se produjera una mejora sustancial de la situación socio-política. Ello produjo el rechazo de numerosos desplazados internos, mayoritariamente bosnios musulmanes, que tenían que abandonar sus residencias temporales en Sarajevo para regresar a pueblos y ciudades de origen que se situaban tras el conflicto en territorios controlados por otros grupos étnicos, ya fueran serbobosnios o bosnio-croatas. El temor a tener que vivir bajo dominación de otro grupo étnico condujo a una ola de construcción informal (e ilegal, a tenor de la normativa vigente) de viviendas entre finales de los años noventa e inicios de la década pasada, y que se propagó por las vertientes de Sarajevo, con el objetivo de encontrar una solución estable en la capital de Bosnia y Herzegovina.

Las principales modalidades de construcción residencial informal constituyeron en elevar el volumen edificatorio ya existente, mediante la construcción de una o dos plantas nuevas, y nuevas viviendas unifamiliares localizadas en áreas suburbanas. Una parte muy significativa de ellas se encuentran en áreas con elevado riesgo geomorfológico debido a su localización en vertientes inestables con pendientes superiores al 20\% (figuras 5 y 6). De hecho, las estimaciones del Instituto de Planificación y Desarrollo Urbano de Sarajevo sugieren que entre 25.000 y 50.000 construcciones ilegales fueron levantadas durante el período de la posguerra ${ }^{3}$ (1996-2003). Estas nuevas viviendas informales fueron en bastantes casos construidas en sitios con pendientes pronunciadas y terrenos inestables, comportando cierta amenaza geomorfológica a estos nuevos asentamientos urbanos como en los barrios de Pofaliči, Aneks o Vraca. Tal urbanización en las vertientes se produjo a pesar de los cambios llevados a cabo por el Instituto de Planificación y Desarrollo Urbano de Sarajevo, que modificó el régimen de regulación de ocupación del suelo urbano en las áreas de las vertientes ante el previsible incremento demográfico fruto de la convergencia del retorno de los ciudadanos de Sarajevo y aquellos que pretendían quedarse en la ciudad tras haberse refugiado en ella. Con dicha modificación

\footnotetext{
${ }^{3}$ Entrevista a técnicos del Instituto de Planificación y Desarrollo del Cantón de Sarajevo.
} 
se requería la elaboración de planes de regulación para permitir la construcción de viviendas, intentando controlar la expansión en las vertientes y ofreciendo, además, las mayores garantías a nivel de servicios.

FIGURA 5

\section{SUBURBANIZACIÓN EN LAS PENDIENTES DEL VECINDARIO DE ANEKS}
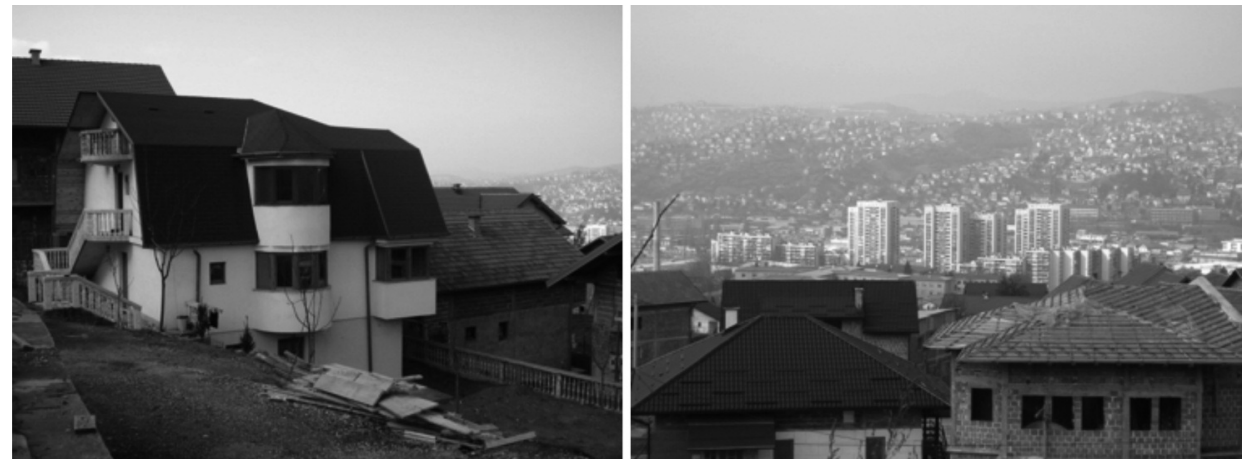

Este proceso de construcción de viviendas informales en las vertientes no era nuevo sino que suponía cierta continuidad con las prácticas llevadas a cabo para durante la época socialista debido a la escasez de viviendas especialmente en los años posteriores a la Segunda Guerra Mundial (Topham, 1990). No fue hasta bien entrada la década de 1970 que la fuerte suburbanización de las primeras cotas de las laderas de los macizos que abrigan Sarajevo empezó a ser visto como una preocupación por parte de las autoridades locales. De ahí que la administración local promoviera el «Programa de Rehabilitación de Áreas Residenciales de las Laderas de la Ciudad» para abordar el desarrollo en esas áreas, consideradas una amenaza potencial para el desarrollo urbano de la ciudad de Sarajevo (Čengić, 2011). Sin embargo, durante el período posbélico y en un contexto en el que los partidos étnico-nacionales pretendían consolidar la territorialización étnica llevada a cabo durante la guerra (Toal y Dahlman, 2006; 2011), la construcción de casas unifiamiliares en las vertientes tuvo un significado muy distinto ya que eran fruto de los desplazamientos forzados y resultado de la imposibilidad o del rechazado de regresar a las áreas de origen. 


\section{FIGURA 6}

ÁREAS CON PENDIENTES SUPERIORES A LOS $20^{\circ} \mathrm{Y}$ NUEVOS PROYECTOS URBANOS DESARROLLADOS DURANTE LA POSGUERRA

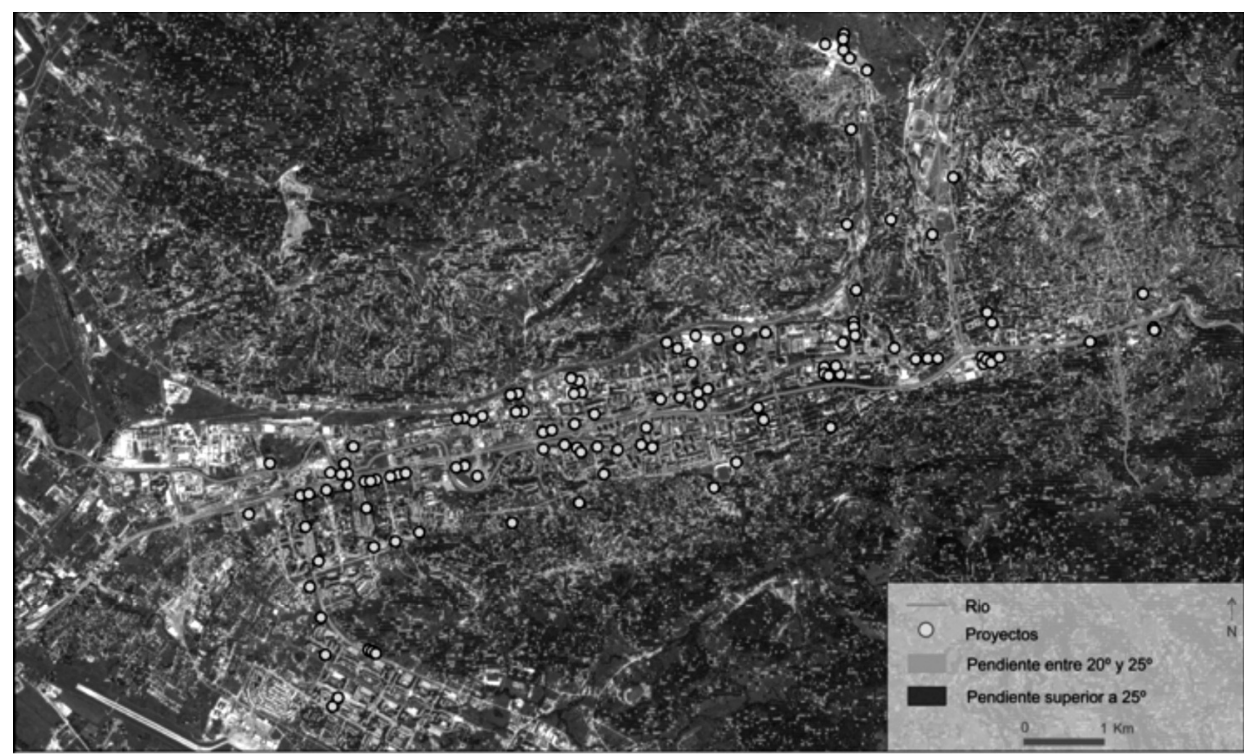

Fuente: a partir de base cartográfica online de acceso libre de Google Earth ${ }^{\circledR} 2014$ y procesamiento de datos a partir de ArcGIS10.

En Sarajevo las construcciones en las laderas no eran producto de la asignación de suelo por parte de las autoridades locales, a diferencia de lo que sucedía en otros municipios de Bosnia en dónde las autoridades locales distribuían el suelo de propiedad social entre personas desplazadas de su propio grupo étnico con el objetivo de consolidar las mayorías étnicas producidas durante la guerra. Debido a que estas prácticas contradecían las políticas de las organizaciones internacionales para fomentar el regreso de las minorías y la reconstrucción de una sociedad multiétnica aplicadas, sólo, tras el apoderamiento del Alto Representante producido en Diciembre de 1997, la Oficina del Alto Representante supervisó las asignaciones de tierra de propiedad social durante el período 1999-2003 (OAR, 1999; 2000). No fue hasta mayo de 2003 cuando la propia Oficina del Alto Representante promulgó una ley del suelo armonizada (la Ley de Construcción de la tierra) en ambas entidades, la FByH y la RS, cediendo de nuevo la gestión del suelo a las municipalidades y 
dividiendo la tierra de propiedad social en suelo estatal o privado sobre la base de si ya había sido desarrollada por un actor privado (Williams, 2013).

La nueva ley fue un paso significativo en los esfuerzos de los actores internacionales para crear un mercado de bienes raíces que llevaba pospuesto desde al menos el año 2000, cuando la Oficina del Alto Representante solicitó un estudio a la USAID en que debía tanto analizarse el estado de los bienes inmuebles en Bosnia y Herzegovina como recomendar su reforma (Rabenshort, 2000). En este sentido, la convergencia de la privatización de la tierra iniciada con la nueva ley del suelo con el aumento de las inversiones, como resultado de la continua presión para la liberalización económica por parte de los diferentes actores internacionales, produjo que a mediados de los años 2000 se produjera el período de máximo desarrollo del mercado inmobiliario en la ciudad después de la guerra.

Un buen ejemplo de esta situación de relativa burbuja inmobiliaria inducida por parte de las organismos internacionales es las numerosas actuaciones inmobiliarias destinadas a la construcción de oficinas y centros comerciales construidos principalmente desde el año 2005 en el distrito central de negocios, el Marijin Dvor, y a lo largo del Bulevar Mese Selimovica, la ancha avenida que discurre paralela al rio Miljacka. Precisamente, la cartografía sobre las áreas inundables de la ciudad de Sarajevo en una cota de 2,5 m por encima del nivel superior de la canalización del curso fluvial indican que un número significativo de estos nuevos proyectos desarrollados en ambos márgenes del río y que han sido construidas en los últimos años presentan una elevada probabilidad de inundación (figura 7).

Estas actuaciones urbanísticas enmarcadas en la promoción de actividades terciarias, a menudo construidas ilegalmente ${ }^{4}$, han jugado un papel central en la reestructuración urbana y en la reorganización espacial de la ciudad de Sarajevo (Martín-Díaz, 2014). Este proceso de densificación urbana a través de la terciarización del suelo urbano (y la consiguiente financialización del proceso de construcción y promoción) se ha concentrado en los barrios surgidos para absorber la elevada migración del campo a la ciudad producida en tiempos del régimen socialista yugoslavo. No cabe duda que la densificación urbana como resultado de las recientes actuaciones urbanísticas englobadas en una clara estrategia de terciarización del espacio urbano y la transición de la ciudad industrial socialista a la ciudad (neoliberal) de servicios pone en serias dificultades la consecución de los objetivos medioambientales y de sostenibilidad urbana fijados en los documentos de planificación estratégica urbana, metropolitana y regional de Sarajevo (KS, 2000).

\footnotetext{
${ }^{4}$ Entrevista a técnicos del Instituto de Planificación y Desarrollo del Cantón de Sarajevo.
} 


\section{FIGURA 7}

\section{NUEVOS PROYECTOS Y ÁREAS POTENCIALES DE INUNDACIÓN EN UN ESCENARIO DE 2,5 M}



\section{CONCLUSIONES}

Las grandes inundaciones ocurridas en mayo de 2014 en Bosnia y Herzegovina, con efectos devastadores en áreas urbanas entre las que se encontraba Sarajevo, han puesto de relieve los riesgos geomorfológicos que están expuestos muchos de estos entornos construidos. El contexto geomorfológico de las zonas centrales de Sarajevo en el valle Miljacka ha condicionado históricamente el desarrollo urbano de la ciudad. La alta presión demográfica sobre Sarajevo en los años posteriores a la guerra de Bosnia (1992-1995) añadió otra capa de complejidad en la gestión del desarrollo urbano. De hecho, anticipando un posible retorno masivo de los refugiados y desplazados internos a la ciudad, el Instituto de Planificación del Desarrollo estableció nuevas herramientas con el fin de controlar el crecimiento urbano en las laderas. La necesidad de aprobar planes de regulación para la urbanización de dichas áreas, sin embargo, no evitó que la expansión urbana desencadenada después de la reposesión de la vivienda por parte de las personas desplazadas se desarrollara de manera significativa en las zonas de gradiente más elevado de la ciudad. 
Por otra parte, las políticas promulgadas por los actores internacionales también tenían implicaciones ambientales significativas, moviendo el entorno construido en Sarajevo hacía una creciente insostenibilidad. Así, las políticas de liberalización del suelo promovidas con la Ley de Construcción de la tierra de 2003 llevaron a una densificación urbana en el centro de Sarajevo con la expansión suburbana en la periferia. Los eventos extremos de precipitación recientes confirman tal dirección de las edificaciones tanto en las zonas urbanas densificadas, así como en los nuevos barrios suburbanos ubicados en pendientes pronunciadas y áreas inundables (por ejemplo Ilidža, figura 4).

Por lo tanto, junto a la limitada incorporación de las visiones ambientales en el resto de la jerarquía de planificación, tanto la creación de un mercado de bienes raíces como la presión demográfica de la posguerra han venido socavando los fundamentos mismos de la agenda de desarrollo sostenible en la ciudad de Sarajevo, como se muestra en la evolución de las edificaciones. En los asentamientos urbanos geomorfológicamente vulnerables, la elaboración e implementación de una estrategia integral sostenible del desarrollo urbano deben ser vistas como una prioridad a pesar de tratarse de procesos sociales y urbanos complejos. En este sentido, la proximidad de la finalización de la Estrategia de Desarrollo cantón de Sarajevo y el Plan Urbano en el 2015 es una oportunidad para repensar una estrategia sostenible social y ambiental más equilibrado, teniendo en cuenta no sólo la particular configuración geomorfológica de la ciudad de Sarajevo, sino también sus especificidades políticas, socio-económicas y simbólicas.

\section{AgradeCimientos}

Para la realización de este trabajo, Jordi Martín-Díaz ha recibido financiación del Programa de Formación del Profesorado Universitario del Ministerio de Educación, para llevar a cabo el proyecto titulado "Geopolítica y reconstrucción urbana en la Sarajevo posbélica y postsocialista" (AP2010-3873). Además Jordi Nofre ha contado con fondos de la Fundaçao para a Ciência e a Tecnología de Portugal (FCT), para el proyecto "Urban Change and Nightlife in Lisbon and Porto: A comparative and transdisciplinary approach" (SFRH/BPD/108458/2015).

Finalmente, este trabajo ha contado con el apoyo logístico del Departamento de Geografía Humana de la Universidad de Barcelona, el Centro de Estudos de Sociologida da Universidade Nova de Lisboa (CESNOVA), y del Centro de Estudos Geográficos do Instituto de Geografia e Ordenamento do Território da Uni- 
versidade de Lisboa (CEG-IGOT/UL). Los autores también agradecen los consejos del Dr. Nihad Čengić (Universidad de Sarajevo) en la elaboración del artículo. Cualquier error o imprecisión es responsabilidad única de los autores.

\section{BiBLIOGRAFÍA}

Adair, A., Berry, J., McGreal, S., Sýkora, L., Parsa, A. G. y Redding, B. (1999): "Globalization of real estate markets in Central Europe", European Planning Studies, 7/3, pp. 295-305.

Adeli, Z. y Khorshiddoust, A. (2011): "Application of geomorphology in urban planning: Case study in landfill site selection", Procedia Social and Behavioral Sciences, 19 , pp. 662-667.

Admir, A., Dženan, B., Alma, H., Emira, H., Mirna, K., Bruno, M., Senka, M., Mersad, O., Bojana, P. A. y Adela, S. (2014): Landscapes of Sarajevo Region, Document de LE: NOTRE Extraordinary Landscape Forum 2014, Faculty of Forestry, University of Sarajevo.

Agencias (2014): "Death toll rises to 43 in Serbia and Bosnia floods", The Telegraph, 18 de Mayo 2014

Albrechts, L. (2004): "Strategic (spatial) planning re-examined", Environmental and Planning B: Planning and design, 31, pp. 743-758.

Alcántara, I. (2000): "Landslides: ¿deslizamientos o movimientos del terreno? Definición, clasificaciones y terminología", Revista Investigaciones Geográficas, UNAM, 41 , pp. $7-25$

Andjelić, N. (2003): Bosnia: The End of a Legacy, Londres, Frank Class Publishers, 228 pp. Baeten, G. (2012): "Neoliberal planning: Does it really exist?", en T. Tasan-Kok, y G. Baeten (eds.), Contradictions of Neoliberal Planning: Cities, Policies, and Politics Springer, Nueva York, Springer, pp. 205-212.

Borén, T. y Gentile, M. (2007): "Metropolitan processes in post-communist states: An introduction", Geografiska Annaler: Series B, Human Geography, 89, pp. 95-110.

Bradley, R. S. y Jones, P. D. (1993): "Little Ice Age summer temperature variations: their nature and relevance to global recent warming trends", The Holocene, 3, pp. 367-376.

Brenner, N. y Theodore, N. (2005): "Neoliberalism and the urban condition", City, 9, pp. 101-107.

Bryson, J. M. y Einsweiler, R. C. (1987): "Strategic Planning: introduction", Journal of the American Planning Association, 53, pp. 6-8.

Čengić, N. (2011): "Remodelling urban meaning - the Sarajevo case", Actas del $4^{\circ}$ Congreso internacional de riesgos y patrimonio moderno (CICOP), Sarajevo.

Champion, T. (2001): "Urbanization, suburbanization, counterurbanization and reurbanization", en R. Paddison (ed.), Handbook of Urban Studies Sage, Londres, Sage, pp. 143-161. 
De Waele, J., Gutiérrez, F., Parise, M. y Plan, L. (2011): "Geomorphology and natural hazards in karst areas: A review", Geomorphology, 134, pp. 1-8.

Dematteis, G. (1998): "Suburbanización y periurbanización. Ciudades anglosajonas y ciudades latinas", en J. Monclu, (ed.), La ciudad dispersa: Suburbanización y nuevas periferias, Barcelona, Centre de Cultura Contemporànea de Barcelona, pp. 17-33.

Diener, A. C. y Hagen, J. (2013): "From socialist to post-socialist cities: narrating the nation through urban space", Nationalities Papers, 41/4, pp. 487-514.

Donia, R. (2006): Sarajevo: A Biography, Londres, Hurst \& Company, 435 pp.

Easterbrook, D. J. (1999): Surface Processes and Landforms, Upper Saddle River, Prentice-Hall, 546 pp.

European Commission (EC) (1993): "Towards Sustainability", Official Journal of the European Communities, C138, p. 5.

Federalna Novinska Agencija (FENA) (2014a): Obrušilo se korito Miljacke kod mosta Ćumurija, 15 de Mayo de 2014.

Federalna Novinska Agencija (FENA) (2014b): Water pumped out from FBiH parliament building Sarajevo, 16 de Mayo de 2014.

Federalna Novinska Agencija (FENA) (2014c): Mještani naselja Svrake prije tri godine bili su upozoreni na klizište!, 21 de Mayo de 2014.

Federalni Zavod za Statistiku (FZS) (2013): "Census of population, households and dwellings in BiH, Preliminary Results by municipalities", FBiH Statistical Bulletin, 195.

French, R. A. y Hamilton, F. E. (1979): The socialist city: spatial structure and urban policy, Nueva York, John Wiley \& Sons, 541 pp.

Golobic, M. y Cof, A. (2010): Knowledge Support for Planning in Transition - Spatial Vulnerability Analysis for Canton Sarajevo, en http://www.kolleg.loel.hs anhalt.de/ landschaftsinformatik/436.html

Gupta, A. y Ahmad, R. (1999): "Geomorphology and the urban tropics: building an interface between research and usage", Geomorphology, 31, pp. 133-149.

Hegedüsa, J. (2007): "The future of re-invented/post-socialist cities in Europe: a reflection on the State of European Cities", Urban Research E Practice, 1/3, pp. 311-318.

Hirt, S. y Stanilov, K. (2009): Twenty years of transition: the evolution of urban planning in Eastern Europe and the former Soviet Union: 1989-2009, Nairobi, UN Habitat.

Hitchcok, W. I. (2003): The Struggle for Europe: The Turbulent History of a Divided Continent 1945-2002, Ontario, Doubleday, 528 pp.

Horner, G. M. (1978): "Kropotkin and the city: The socialist ideal in urbanism", Antipode, 10/11, pp. 33-45.

Hrvatović, H. (2005): Geological guidebook through Bosnia and Herzegovina, Sarajevo, Geological Survey of Bosnia and Herzegovina, 156.

Huang, J. y Mu, G. (2000): "Urban geomorphology", Progress in Physical Geography, 24, pp 451-452. 
Hyman, L. (1999): "The Effects on Labor of Post-Industrial Globalization: A Comparison of Marxist and Non-Marxist Paradigms, Journal of Economics, 3/1, p. 3, en http://digitalcommons.iwu.edu/uauje/vol3/issl/3.

Jennings, J. N. (1985): Karst Geomorphology, Oxford, Basil Blackwell, 293 pp.

Jukic, E. (2014a): Bosnia Flooding Causes Landslides Destroys Homes, BIRN, Sarajevo 15 May 14.

Jukic, E. (2014b): Bosnia Hopeful Ahead of Donors Conference, Sarajevo, BIRN, 15 July 2014.

Kääb, A., Huggel, C., Paul, F, Wessels, R., Raup, B., Kieffer, H. y Kargel, J. S. (2003): "Glacier monitoring from ASTER imagery. Accuracy and applications", EARSeL eProceedings 2-Observing our cryosphere from space, pp. 43-53.

Kamp, U., Bolch, T., y Olsenholler, J. (2005): "Geomorphometry of Cerro Sillajhuay, Chile/Bolivia: comparison of DEMs derived from ASTER remote sensing data and contour maps", Geocarto International, 20, pp. 23-34.

Kanton Sarajevo (KS) (2000): Sarajevo Development Strategy until the year 2015, Sarajevo, Ministry of reconstruction, development and environmental protection.

Luterbacher, J., Dietrich, D., Xoplaki, E., Grosjean, M. y Wanner, H. (2004): "Extreme Seasonal and Annual Temperature Variability, Trends and Extremes Since 1500", Science, 3, pp. 1499-1503.

Luterbacher, J., Xoplaki, E., Dietrich, D., Jones, P. D., Davies, T. D., Portis, D., Gonzales-Rouco, J. F., Von Storch, H., Gyalistras, D., Casty, C. y Wanner, H. (2002): "Extending North Atlantic Oscillation reconstructions back to 1500", Atmospheric Science Letters, 2, pp. 114-124.

Maier, K. (1994): "Planning and education in planning in the Czech Republic", Journal of Planning Education and Research, 13, pp. 263-269.

Majstorovic, Z., Tais, M., Voloder, M. y Zulum, D. (2006): The correlation between air pollution and air temperature in Sarajevo Valley (1975-2006), Sarajevo, Federal Hydro Meteorological Institute.

Martín-Díaz, J. (2014): "Urban restructuring in post-war contexts: the Sarajevo case", Hungarian Geographical Bulletin, 63/3, pp. 303-317.

Martín-Díaz, J. (2016): Geopolitics and urban reconstruction in post-socialist Sarajevo, tesis doctoral.

Masser, I. (1992): "Learning from Europe", Journal of the American Planning Association, 58, pp. 3-8.

Oficina del Alto Representante (OAR) (1998): Sarajevo Declaration and Implementation Reports, http://www.ohr.int/ohr-dept/rrtf/key-docs/sa-docs/ (Fecha de consulta: 15/4/2014).

Oficina del Alto Representante (OAR) (1999): Decision extending until 30 June 2000 the Decision on certain types of socially-owned land, http://www.ohr.int/deci sions/plipdec/default.asp?content_id=210 (Fecha de consulta: 25/4/2014).

Oficina del Alto Representante (OAR) (2000): Decision on re-allocation of socially owned land, superseding the 26 May 1999 and 30 December 1999 Decisions, 
http://www.ohr.int/decisions/plipdec/default.asp?content_id=213 (Fecha de consulta: 25/4/2014).

Papadopoulou-Vryniotia, K., Bathrellos, G. D., Skilodimoua, H., Kavirisb, G. y Makropoulos, K. (2013): "Karst collapse susceptibility mapping considering peak ground acceleration in a rapidly growing urban area", Engineering Geology, 158, pp. 77-88.

Peck, J., Theodore, N. y Brenner, N. (2009): "Neoliberal Urbanism: Models, Moments, Mutations", SAIS Review of International Affairs, 29/81, pp. 49-66.

Rabenshort, C. S. (2000): The real estate market in Bosnia-Herzegovina: Current status and recommendations for reform, Sarajevo, USAID.

Sailer-Fliege, U. (1999): "Characteristics of post-socialist urban transformation in East Central Europe", GeoJournal, 49/1, pp. 7-16.

Sarajevo Times (ST) (2014): Two and a Half Meter is Miljacka's Water Level, FENA, 14 de Mayo de 2014.

Smith, A. y Rochovká, A. (2007): "Domesticating neo-liberalism: Everyday lives and the geographies of post-socialist transformations", Geoforum, 38/6, pp. 1163-1178.

Stecchi, F., Mancini, F., Ceppi, C. y Gabbianelli, G. (2012): "Vulnerability to ground deformation phenomena in the city of Tuzla (BiH): a GIS and multicriteria approach", Natural Hazards, 64, pp. 2153-2165.

Stenning, A., Smith, A., Rochovská, A. y Świątek, D. (2010): Domesticating neo-liberalism: Spaces of economic practice and social reproduction inpost-socialist cities, Londres, Willey-Blackwell, 320 pp.

Sweeting, M. M. (1973): Karst Landforms, Londres, Macmillan, 362 pp.

Swyngedouw, E., Moulaert, F. y Rodriguez, A. (2002): "Neoliberal Urbanization in Europe: Large-Scale Urban Development Projects and the New Urban Policy", Antipode, 34 , pp. 542-577.

Sýkora, L. y Stanilov, K. (2014): "The Challenge of Postsocialist Suburbanization", en K. Stanilov y L. Sýkora, L. (eds.), Confronting Suburbanization: Urban Decentralization in Postsocialist Central and Eastern Europe, Chichester, John Wiley \& Sons, Ltd.

Szelenyi, I. (1996): "Cities under Socialism - and after", en G. Andrusz, M. Harloe e I. Szelenyi, [eds.], Cities after socialism, Oxford, Blackwell, pp. 286-317.

Tasan-Kok, T. (2012): "Introduction: Contradictions of Neoliberal Urban Planning", en T. Tasan-Kok y G. Gaeten (eds.), Contradictions of neoliberal urban planning. Cities, Policies and Politics, Nueva York, Springer, pp. 1-19.

Theodore, N., Peck, J. y Brenner, N. (2011): "Neoliberal Urbanism: Cities and the Rule of Markets", en G. Bridge y S. Watson (eds.), The New Blackwell Companion to the City, Oxford, Wiley-Blackwell.

Toal, G. y Dahlman, C. (2006): "The 'West Bank of the Drina': Land Allocation and Ethnic Engineering in Republika Srpska", Transactions Institute of British Geographers, 31, pp. 304-322.

Toal, G. y Dahlman, C. (2011): Bosnia remade, Nueva York y Oxford, Oxford University Press, 463 pp. 
Topham, S. (1990): "Housing policy in Yugoslavia", en J. Sillience (ed.), Housing policies in Eastern Europe and the Soviet Union, Nueva York, Routledge, pp. 6-57.

Tosics, I. (2005): "City development in Central and Eastern Europe since 1990: The impacts of internal forces", en F. E. I. Hamilton, K. Dimitrovska y N. Pitchler-Milanovic (eds.), Transformation of cities in central and Eastern Europe: Towards globalization, Tokyo, United Nations University Press, pp. 44-78.

Tsenkova, S. (2007): "Urban futures: Strategic planning in post-socialist Europe", en K. Stanilov (ed.), The Post-Socialist City, Urban Form and Space Transformations in Central and Eastern Europe after Socialism, Dordrecht, Springer, pp. 447-472.

Tsenkova, S. y Nedović-Budić, Z. (2006): The urban mosaic of post-socialist Europe: space, institutions and policy, Heidelberg, Physica-Verlag, 389 pp.

UNHCR (1999): UNHCR Global Appeal 1999, Bosnia and Herzegovina, UNHCR Fundraising Reports, http://www.unhcr.org/3eaff43e9.html (Fecha de consulta: $1 / 4 / 2014$ ).

Wanner, H., Beck, C., Brádzil, R., Casty, C., Deutsch, M., Glaser, R., Jacobeit, J., Luterbacher, J., Pfister, C., Pohl, S., Sturm, K., Werner, P. C. y Xoplaki. E. (2004): "Dynamic and socioeconomic aspects of historical floods in central Europe", Erdkunde, 58, pp. 1-16.

Williams, R. (2013): "Post-conflict land tenure issues in Bosnia: Privatization and the politics of reintegration of displaced", en J. Unruh y R. Williams (eds.), Land and Post-Conflict Peacebuilding, Nueva York, Routledge, pp. 145-176.

Xoplaki, E., Maheras, P. y Luterbacher, J. (2001): "Variability of climate in meridional Balkans during the periods 1675-1715 and 1780-1830 and its impact on human life", Climatic Change, 48, pp. 581-615.

Fecha de recepción: 19 de diciembre de 2014.

Fecha de aceptación: 16 de julio de 2015. 